\title{
COLLABORATION SCRIPTS - A CONCEPTUAL ANALYSIS
}

This is a post-print of an article submitted for consideration in the Educational Psychology Review (C) 2006 Springer Verlag.

Personal use of this manuscript is permitted. Permission from Springer Verlag must be obtained for any other commercial purpose.

This article may not exactly replicate the published version, due to editorial changes and/or formatting and corrections during the final stage of publication. Interested readers are advised to consult the published version which can be found at:

$\underline{\text { http://www.springerlink.com/content/j740400g58410x51/ }}$

doi: $\{10.1007 / \mathrm{s} 10648-006-9007-2\}$

Please refer this manuscript as:

Kollar, I., Fischer, F., \& Hesse, F. W. (2006). Collaboration scripts - a conceptual analysis. Educational Psychology Review, 18(2), 159-185. 
Running Head: COLLABORATION SCRIPTS

Collaboration Scripts - A Conceptual Analysis

Ingo Kollar, Frank Fischer, and Friedrich W. Hesse

Knowledge Media Research Center, Tübingen (Germany)

Corresponding author:

Ingo Kollar, M.A.

Knowledge Media Research Center

Konrad-Adenauer-Strasse 40

D-72072 Tübingen/Germany

phone: $\quad++49-7071$ 979-245

fax: $\quad++49-7071979-100$

email: i.kollar@iwm-kmrc.de 


\begin{abstract}
This article presents a conceptual analysis of collaboration scripts used in face-to-face and computer-mediated collaborative learning. Collaboration scripts are scaffolds that aim to improve collaboration through structuring the interactive processes between two or more learning partners. Collaboration scripts consist of at least five components: (a) learning objectives, (b) type of activities, (c) sequencing, (d) role distribution, and (e) type of representation. These components serve as a basis for comparing prototypical collaboration script approaches for face-to-face vs. computer-mediated learning. As our analysis reveals, collaboration scripts for face-to-face learning often focus on supporting collaborators in engaging in activities that are specifically related to individual knowledge acquisition. Scripts for computer-mediated collaboration are typically concerned with facilitating communicativecoordinative processes that occur among group members. The two lines of research can be consolidated to facilitate the design of collaboration scripts, which both support participation and coordination, as well as induce learning activities closely related to individual knowledge acquisition and metacognition. In addition, research on collaboration scripts needs to consider the learners' internal collaboration scripts as a further determinant of collaboration behavior. The article closes with the presentation of a conceptual framework incorporating both external and internal collaboration scripts.
\end{abstract}

Keywords:

computer-supported collaborative learning, scripts, collaboration scripts, scaffolding. 


\section{Introduction}

Research on collaborative learning has repeatedly demonstrated that learners often do not collaborate well spontaneously (Cohen, 1994). For example, they tend not to participate equally (Cohen and Lotan, 1995), often engage only in low-level argumentation (Bell, 2004), and rarely converge on a comparable level of knowledge acquisition (Fischer et al., 2002). There is a need for instructional support that guarantees a higher quality of both collaborative learning processes and individual learning outcomes. Such instructional support has been described and analyzed more systematically as scaffolding (e.g., Quintana et al., 2004; Pea, 2004; Reiser, 2004; Sherin et al., 2004; Tabak, 2004). Derived from the Vygotskyan concept of the Zone of Proximal Development (Vygotsky, 1992), scaffolding is seen as a way to support learners as they accomplish tasks that they would not be able to accomplish on their own (Wood et al., 1976). Originally addressing parent-child interactions, the term scaffolding has also been used to describe artifact-based instructional support (e.g., Quintana et al., 2004). With respect to collaborative learning, at least two classes of scaffolds can be distinguished: (a) scaffolds that provide support on a conceptual level and (b) scaffolds that provide support related to the interactive processes between the collaborators. Scaffolds that provide learners with conceptual support relevant to the contents of a task include, for example, questions or prompts that guide learners in discussing a specific aspect of a physics problem such as "What is the relationship between force and motion?" or "If ball A hits ball B with the speed of X, what is the resulting speed of ball B?" Scaffolds that structure the interactive processes of collaborative learning shape collaboration by specifying different roles and associated activities to be carried out by the collaborators. For example, learners are asked to explain the contents of a text and to critique contributions of their learning partners at specific points in the learning process. Especially in 
research on computer-supported collaborative learning (CSCL), such scaffolds have been called collaboration scripts (e.g., Dillenbourg, 2002; Rummel and Spada, 2005; Weinberger et al., 2005). They describe an instance of socio-cognitive structuring (Ertl et al., in press).

Collaboration scripts have been used to structure both face-to-face (e.g., O'Donnell and Dansereau, 1992; Palincsar and Brown, 1984) and computer-mediated collaboration (e.g., Dillenbourg, 2002; Rummel and Spada, 2005; Weinberger et al., 2005). However, given the variety of existing collaboration script approaches, the term lacks conceptual precision. In this article, we analyze systematically previous research on collaboration scripts. We first work towards a definition of collaboration scripts by identifying central conceptual components that are shared among different approaches. We then compare prototypical collaboration script approaches used for either face-to-face or computer-mediated learning on the basis of these conceptual components to detect commonalities, differences, and shortcomings of the two lines of research. Based on the identified deficits, we develop a conceptual framework that describes how collaborators and collaboration scripts interact during collaboration tasks. We believe that this framework can be used to inform both the design of collaboration script approaches and future theory building and to trigger subsequent empirical research on collaboration scripts.

In sum, the article focuses on the following questions:

(1) What are the central conceptual components of collaboration scripts?

(2) Based on these components, what are the commonalities, differences, and shortcomings of collaboration scripts developed for face-to-face and computer-mediated learning?

(3) What can be derived from this comparison with respect to design, theory building, and empirical research on collaboration scripts? 


\section{Central Conceptual Components of Collaboration Scripts}

To answer research question 1 concerning the central conceptual components of collaboration scripts, we start by examining the original meaning of the term script in cognitive psychology. Schank and Abelson (1977) used the term to refer to culturally shared knowledge about the world that provides information about conditions, processes, and consequences of particular everyday situations. In this perspective, scripts consist of a number of variables (or slots) for persons or objects playing prominent roles in such situations. A script provides individuals with knowledge about how these variables function within the course of action represented in the particular script. Furthermore, the script provides individuals with information about appropriate actions within the particular situation and helps them better understand the everyday situations they are involved in. This results in enhanced information processing and in a reduced need for coordination between the actors. Schank and Abelson (1977) explain the meaning of scripts by offering the "restaurant script" as an example. This script specifies that the guest enters the restaurant, signals the waiter, asks for the menu, chooses one item from it, waits for the meal to be brought to his table, etc. Using this script, a listener is able to understand short information sequences like "Martin went to a restaurant and ate a beef steak". By possessing an adequate, culturally shared script and knowing its implications, the recipient has the knowledge necessary to fill in any gaps in the information sequence. In the case of the restaurant script, the recipient is, therefore, able to conclude that Martin entered the restaurant, signaled the waiter to have a look at the menu, chose one menu item, namely the beef steak, ordered it, waited until it was prepared, etc.

Cognitive psychology typically views scripts as highly specific memory structures that remain relatively fixed in situations in which the script is activated. This is especially true for 
approaches, which aim at developing computational models of cognitive systems that work in accordance with a prespecified script. For such models to function properly, the individual steps and activities of the script must be well-defined and comprise detailed rules (see Schank, 1999). For example, in the restaurant script, first taking a seat and then choosing a meal from a menu are activities that are constitutive for the activity "going to a restaurant" and are only alterable under exceptional circumstances (e.g., when all tables are occupied and guests have to wait at the bar). In contrast, collaboration scripts can vary in the degrees of freedom they attribute to learners to structure their collaboration. In a rather open version, they can provide a frame of reference in the form of a scenario (e.g., giving the global instruction to critique each other's contribution) without giving further instruction on what form the process of critiquing should assume. Learners are then relatively free to choose and perform appropriate activities (for example, re-read a text, ask thought-provoking questions, provide counterevidence, etc.). In scenarios using more restrictive collaboration scripts, learners are severely constrained in the specific activities they can choose from and how they perform them (e.g., a computer interface that demands a specific on-screen action be performed by a participant before displaying the next screen).

Although the usage of the term script diverges between cognitive psychology and educational psychology, at least five similarities can be detected, which might help derive a preliminary definition of what collaboration scripts are. First, both scripts used in cognitive psychology (which are viewed as individual cognitive structures) and in educational psychology (which are known as collaboration scripts) pursue specific objectives. According to Schank and Abelson (1977), scripts enhance both understanding and recall (on an individual level; e.g., Nuthall, 2000) and promote the coordination of activities in a particular situation (on a group 
level). Similarly, collaboration scripts are goal-oriented in the sense that specific approaches help learners engage in smooth collaboration processes and reach specific (learning) objectives.

Second, both cognitive scripts and collaboration scripts engage individuals in specific activities. In the restaurant script, these activities include entering the restaurant, waiting to be seated, following the waiter to a table, etc. In collaboration script approaches, such activities might be summarizing, questioning or explaining. The specificity of the script instructions can range from merely naming the activities learners are supposed to perform (e.g., "give an argument") to the specification of individual substeps of the activity (e.g., "give evidence", "state a claim", "give a reason" as substeps of developing an argument; see Kollar et al., 2005). Each of the imposed (sub-)activities can also be presented in a more or less structured way. Although some scripts provide learners with a lot of freedom as to how to perform a specific activity, others do not. Activities should, however, be in accordance with the pre-defined objective of the script, regardless of whether they are broken down into scripted substeps or whether there are constraints on how the activities can be performed.

Third, sequencing plays an analogous role in scripts from the perspectives of cognitive and educational psychology. In both cases, the sequencing in the script not only specifies which activities learners should perform, but also when they should perform them. In the restaurant script, the script provides actors with sequential knowledge about first entering the restaurant, then waiting to be seated (in the U.S. - but not in Europe), following the waiter to a table, and so on. Collaboration scripts also specify or imply which activities collaborators should perform and in what order, for example, first reading a text and then summarizing it (see O'Donnell and Dansereau, 1992). 
Fourth, cognitive scripts and collaboration scripts specify and distribute roles among the participating individuals. The restaurant script specifies that the waiter brings the menu and the guest chooses a meal from it. Similarly, collaboration scripts distribute roles among the collaborating partners, for example, an explainer and a commentator role. Distributing collaboration roles helps support the collaborating partners in approaching the task from multiple perspectives. This, in turn, helps learners consider problems from various viewpoints (Spiro et al., 1991) and reduces the danger of acquiring inert knowledge (Renkl et al., 1996). In both cognitive and collaboration scripts, role distribution, however, is not always made explicit. Sometimes, the activities that one participant is supposed to conduct define his or her role without explicitly naming this role. For example, playing a commentator role is automatically implied by having learners critique other learners' contributions.

Fifth, scripts can vary in the type of representation through which specific instructions are presented to the learners. Cognitive scripts are internal (mental) representations about courses of action in particular situations. Collaboration scripts are external representations that are presented textually (e.g., King, 1998), as graphical representations (Pfister and Mühlpfordt, 2002), or orally (e.g., Palincsar and Brown, 1984). There is evidence that different representations have differential effects on learning, which may, in turn, interact with learner characteristics (e.g., Mayer, 2001; Plass et al., 1998).

In summary, a comparison between cognitive scripts and collaboration scripts reveals several parallels that can help define what collaboration scripts are. We identified five components as being pivotal for answering the first research question: "What are central conceptual components of collaboration scripts?" These components are: (1) learning objectives, (2) type of learning activities, (3) sequencing of activities, (4) role distribution mechanisms, and 
(5) type of representation (see table 1). As a working definition, a collaboration script can be described as an instructional means that provides collaborators with instructions for task-related interactions, that can be represented in different ways, and that can be directed at specific learning objectives. These objectives can be reached by inducing different kinds and sequences of activities, which are implicitly or explicitly clustered to collaboration roles. Scripted activities can be broken down into individual acts that together form a larger activity, and scripts can vary with respect to how much structure they provide.

Having defined the term collaboration script, we next review existing collaboration script approaches for face-to-face vs. computer-mediated learning by applying these five conceptual components (research question 2). By comparing approaches from these two lines of research, we aim to identify areas of convergence and divergence and thus pinpoint areas in which both lines of research can fruitfully inform the other concerning the appropriate design of collaboration scripts and theory building. We further identify common research deficits that might inform future empirical research in the field.

INSERT TABLE 1 ABOUT HERE

\section{Collaboration Scripts for Face-to-Face Learning}

Over the last twenty years, research has documented ways to improve collaborative learning. In the following section, we present four prototypical collaboration script approaches developed for face-to-face learning. The collaborative learning programs reviewed improve the interaction between collaboration partners and boost collaborative learning. All the approaches are empirically based or have triggered substantial research. They are germane to different group sizes - from dyads to whole classes. The approaches under consideration are $\underline{\text { Scripted }}$ Cooperation (e.g., O’Donnell and Dansereau, 1992), ASK to THINK - TEL WHY (e.g., King, 
1997), Structured Academic Controversy (Johnson and Johnson, 1994), and Reciprocal Teaching (Palincsar and Brown, 1984).

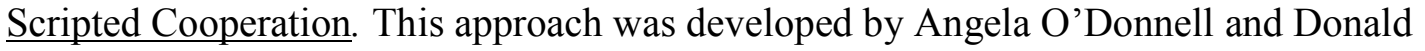
Dansereau and their colleagues and triggered a large research agenda (for an overview, see O’Donnell, 1999). Several variants of Scripted Cooperation were subsequently developed and empirically tested (e.g., Larson et al., 1985; O’Donnell et al., 1987; Rewey et al., 1992). The original MURDER script (the acronym stands for "mood", “understand”, "recall”, “detect", "elaborate", "review" and describes its sequence) involves the interaction between two partners learning from a text. First, the experimenter or the learners themselves split the text up into paragraphs. Then, each learner reads the first passage individually. After that, the partners put the text aside and engage in different roles: One plays the recaller, whose task is to recall the text information as completely as possible. The other partner plays the listener and tries to detect and correct misconceptions and identify omissions. After that, the partners elaborate jointly on ways to make the text content more memorable. They can accomplish this by connecting the information with their own prior knowledge, for example, by drawing comparisons or links to other topics. Once the dyad has worked through the first text passage in this manner, the next segment is read and roles are switched. The script instructions can be presented to the learners in different ways. Lambiotte et al. (1987) provided the instructions in written format after each text paragraph. Larson et al. (1984) did not provide learners with written instruction during collaboration, but instead trained learners on the correct application of the script instructions prior to the actual collaborative learning phase. Hythecker et al. (1988) state that the usual training time is about one hour. 
The objectives of the MURDER script are twofold. First, learners are supposed to acquire knowledge about text content. Second, they are supposed to acquire text-learning strategies. These strategies include cognitive skills, such as explaining, and metacognitive skills, such as monitoring. In accordance with these objectives, Scripted Cooperation increases learners' engagement in cognitive and metacognitive activities. As an example of cognitive activities, learners are supposed to engage in explaining. As an example of metacognitive activities, the collaboration script requires learners to engage in monitoring. To ensure that learners perform these activities effectively, they are often supposed to practice them first during a training phase. Sequencing of the activities is regulated by the different phases introduced in the MURDER script. First, both partners have to read a text passage. Next, learner A summarizes the text and learner B tries to detect and correct misconceptions and identify omissions. Then, the learning partners elaborate on the text content to make it more memorable. Finally, they read the next paragraph. This sequence is fixed and cannot be changed by the learners. The script also specifies and distributes collaboration roles. After having read a text passage, one learner has to play the recaller, while the other plays the listener. These roles are switched several times during the learning process. The representation type of the MURDER script varies between empirical studies. In some instances, the instructions are presented and internalized before the actual collaboration phase, so that the collaboration is guided by the learners' mental representations of the MURDER script. In other studies, the instructions are written on the same sheets of paper that contain the text the learners are reading.

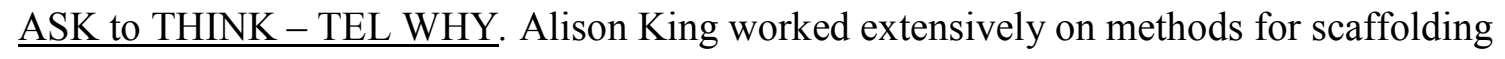
collaboration, with a focus on supporting peer questioning. She developed a peer-tutoring approach for classrooms to support knowledge construction in dyads or in larger groups of 
learners (King, 1997, 1998, 2002). The ASK to THINK - TEL WHY model distributes structured reciprocal tutoring roles (questioner vs. explainer) among the learners and attaches specific activities to these roles. These activities are initially introduced by the teacher, who models them in class before the learners apply them in their subsequent collaboration (the training time is about 160 minutes spread over four school lessons; King, 1997). There are three main groups of activities: (a) specific question types that the learner in the questioner role asks during collaboration (review questions, thinking questions, probing questions, hint questions, and selfmonitoring questions), (b) elaborative explanations that the learner in the explainer role creates in reacting to those questions (including answering the "why" and "how" of the question, as well as establishing links to one's own prior knowledge - and to that of the partner - rather than merely describing objects), and (c) communicative skills, such as listening attentively, providing sufficient thinking time, giving evaluative feedback, etc. After reading a text or listening to a class presentation, learners individually create and write down two review questions and two thinking questions. After that, the learning partners determine who plays the questioner and who plays the explainer first. The questioner then asks one review question (e.g., "What does ... mean?") to activate the explainer's knowledge about the topic at hand. If the explainer fails to answer the question, the questioner then asks probing questions (e.g., "Tell me more about...”) or hint questions (e.g., "Have you thought about...?"). If the review question is answered correctly, the questioner proceeds by asking thinking questions (e.g., "What do you think would happen to ... if ... happened?”). When appropriate, the questioner asks self-monitoring questions (metacognitive questions) that help the explainer make his or her learning process explicit and monitor it effectively. Throughout this process, learners are supposed to follow the communication rules mentioned above (giving appropriate thinking time, etc.). Learners are 
equipped with prompt cards that remind them to follow the sequence of question types. These prompt cards contain question starters for each question type and descriptions of what elaborated explanations are and what communication rules to apply during collaboration. After one complete cycle, the questioner and explainer roles are switched.

The objectives of the ASK to THINK - TEL WHY approach are to support learners in their acquisition and comprehension of information presented in texts or oral presentations, as well as in developing the skills necessary to process the content. This includes cognitive skills related to asking questions, metacognitive skills related to monitoring, and communication skills related to giving enough thinking time. Accordingly, the activities can be grouped into cognitive, metacognitive, and communicative activities. In addition, these activities are explicitly sequenced: The learner in the questioner role asks certain question types in a pre-specified order. The script further prescribes an explicit distribution of collaboration roles. It specifies that one of the collaborators plays the questioner while the other plays the explainer. A role switch is also included. With respect to representation type, the model relies on (a) the teacher orally modeling the instructions (i.e., auditive representation) and (b) the prompt cards containing written reminders of the activity type (i.e., written representation).

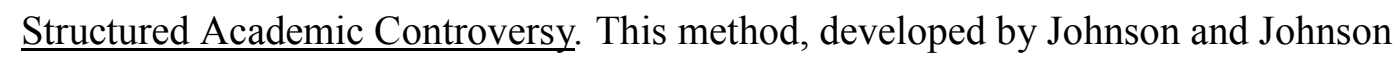
(1994), involves groups consisting of four learners. Within these groups, dyads are created and assigned to opposing positions on a specific topic. The learning material is distributed between the two pairs and the dyads are instructed to make any information in their own material available to the other dyad when it might support their position. Pairs then develop their position and present their arguments to the other dyad. During this presentation, learners exchange thoughts and information, possibly create counterarguments to the other dyad's arguments and 
discuss the rationale of their group's approach. In this step, the discussion can be led relatively freely. However, the teacher encourages learners to abide by certain rules of constructive controversy, which they are introduced to before collaboration. The listeners are instructed to listen to the arguments as carefully as possible because they will later have the task of supporting their counterdyad's position. In the next step, a role switch indicates that the two dyads must adopt and present the position they have just tried to rebut. After that, the positions are dropped and all four learners are instructed to seek a synthesis of their discussion by writing a joint position statement. This position is to be presented to the class later on. Johnson and Johnson (1994) emphasize that training on social and interpersonal skills should precede the controversy, including “confirming others' competence while disagreeing with their positions and challenging their reasoning (being critical of ideas, not people)" or "first bringing out all the ideas and facts supporting both sides (differentiating the differences between positions) and then trying to put them together in a way that makes sense (integration of ideas)" (p. 80). The teacher presents these instructions prior to collaboration and the learners practice them. The instructions also appear on the learners' instructional sheets that they have at their disposal during collaboration.

Structured Academic Controversy aims at two kinds of objectives, namely to support learners (a) in an acquisition of knowledge about the topic at hand and (b) in an acquisition of debating skills. Therefore, the script induces relevant cognitive and metacognitive learning activities. Cognitive activities include generating arguments, explaining arguments, and generating counterarguments. Metacognitive activities include taking the opposing perspective or paraphrasing what someone said if it is not clear. Structured Academic Controversy further prescribes sequences of the induced learning activities. Learners are informed about the different collaboration phases: the development and presentation of a position, discussing the presentation, 
reacting to counterarguments on that position, adopting the other dyad's position, and finding a synthesis. Within the individual steps, learners are free in how they choose to carry out these activities because no further support is provided on how to create arguments or how to rebut a position. The approach further distributes collaboration roles among the learners and gives instructions on what to do when adopting these roles. The script has one dyad present its position while the other dyad listens. Similarly, roles are distributed in subsequent phases. The script also specifies a role switch. Apart from naming the induced activities associated with these roles, there is no further support specifying how to act in these roles. The script's representation type includes an auditive representation of the teacher's oral presentation of the strategies prior to collaboration and written reminders on the instructional materials that provide rough guidelines rather than specific steps and substeps.

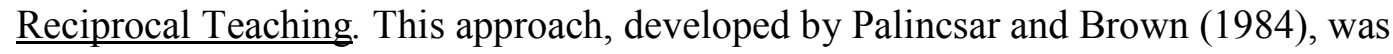
designed to support reading comprehension in beginning readers and children with poor reading comprehension abilities. At the core of this approach are four reading strategies that the teacher introduces to the class. These strategies are questioning, clarifying, summarizing, and predicting. After the teacher has modeled the correct application of the strategies, learners are divided into small groups of variable size. They then work to apply the strategies when reading new text passages, thereby rotating the teacher role among them. The adult teacher then takes on a coaching role and, in the ideal case, eventually abandons the teaching role so that the learners can take it over. The four strategies form a broad framework in which discussion about the text takes place: At first, the student in the teacher role asks questions concerning the content of the text. Next, the group discusses these questions and formulates further questions before the student in the teacher role summarizes the most essential parts of the text passage. If someone 
does not agree with that summary, all learners reread the passage and discuss the emerging summaries until they have agreed upon one variant. After that, learners make predictions about the next text passage. The duration of the intervention can span several weeks.

The objectives of Reciprocal Teaching have both a cognitive and a metacognitive dimension: From a cognitive perspective, learners are supposed to be supported in comprehending text content. From a metacognitive perspective, they are supposed to acquire comprehension-monitoring skills. Consequently, the induced learning activities are part cognitive and part metacognitive. Cognitive learning activities include questioning and clarifying; metacognitive activities include summarizing and predicting. Learning the correct application of these activities is an iterative process guided by the adult teacher, who is supposed to assume a coaching function rather than give detailed instructions on how the activities should be conducted. The activities are further performed in a specific sequence: First, the student in the teacher role asks questions about the text, which are then clarified by the group. After that, learners generate a summary. At the end of a cycle, they make predictions about how the text continues. In addition, the task of leading the discussion rotates between the learners.

Collaboration roles are distributed in this manner: At any time during collaboration, one learner plays the role of the discussion leader, while his or her co-learners stay in their natural pupil role. A role switch prescribes that each learner is supposed to play the discussion leader at least once. With regard to the representation type, it appears that the script instructions are not always externally represented during collaboration. Before collaboration, the teacher presents the learning activities and their sequence orally via modeling and can re-introduce them whenever appropriate during collaboration. However, as long as the learners apply the script appropriately, the teacher does not interfere and re-introduce the script instructions again. 


\section{Comparing Collaboration Scripts for Face-to-Face Learning}

Although the selected approaches cover a variety of tasks, learning settings, and group sizes, they do exhibit certain commonalities. The presented approaches typically target two classes of objectives: cognitive objectives and metacognitive objectives. With respect to cognitive learning objectives, all approaches support learners in gaining knowledge about the text or task, as well as in acquiring elaborative learning strategies, such as questioning or explaining. The presented approaches also focus on promoting the acquisition of metacognitive skills like monitoring, which can be considered higher-order learning strategies (Rosenshine and Meister, 1994). Empirical research indicates that these interventions have helped learners achieve their particular learning objectives (e.g., O’Donnell, 1999; King, 1998; Johnson and Johnson, 1994; Palincsar and Brown, 1984).

The presented approaches aim to promote different learning activities: For example, the MURDER script has learners engage in activities such as summarizing or monitoring, whereas Johnson and Johnson's (1994) approach is more directed towards arguing and debating. From a more abstract perspective, the learning activities can be labeled as cognitive and/or metacognitive activities. However, there are differences with respect to how specific the instructions concerning these activities are. For example, the ASK to THINK - TEL WHY approach structures the activities on a rather detailed level by requiring learners to complete question prompts. Reciprocal Teaching gives rather general directives on how to engage successfully in clarifying, questioning, summarizing, and predicting. The activities induced in Structured Academic Controversy are more implied than explicitly stated. By focusing on promoting learners to engage in elaborative and metacognitive activities, collaboration scripts for face-to-face learning tend to adopt an individual learner perspective: They are primarily 
concerned with augmenting the learning outcomes of all individual learners through their participation in the collaborative learning experience. In other words, collaboration is primarily seen as a means to support individuals in their acquisition of knowledge. Group-building and group-supporting processes, such as communicating or coordinating, are not the primary focus of this perspective. However, group processes are touched upon in ASK to THINK - TEL WHY, Structured Academic Controversy, and Reciprocal Teaching, for example, by demanding that learners provide each other with enough thinking time.

With respect to their sequencing procedures, the four approaches are highly specific. In Scripted Cooperation and ASK to THINK - TEL WHY, learners are provided with specific directives concerning when to engage in which learning activity. To a lesser extent, this is also true for Reciprocal Teaching and for Structured Academic Controversy. Reciprocal Teaching sequences the four learning strategies that the whole class is supposed to employ and Structured Academic Controversy puts four collaboration phases in a fixed order. However, the concrete activities that learners are allowed to demonstrate in these phases are less strictly prescribed than in Scripted Cooperation and ASK to THINK - TEL WHY.

In terms of role distribution, there are fewer observable differences among the four approaches. They all regulate explicitly what roles are distributed between the learning partners. Moreover, all approaches include an explicit role switch to have each learner experience the benefits of each collaboration role and the associated learning activities. Reciprocal Teaching is the approach which gives learners the most freedom of choice regarding which role they want to assume. The learners themselves decide whether they want to adopt the teacher's role. In the other approaches, there is less opportunity for the learners to control their involvement in one or 
another role because the scripts provide explicit regulations specifying that each learner must engage in each of the corresponding roles.

Regarding the representation type of the presented collaboration scripts, it is common for the specific instructions to be presented orally by a teacher or an experimenter and practiced by the learners before collaboration. These trainings can take up to several hours, as is the case in Scripted Cooperation or Reciprocal Teaching. During the actual collaboration phase, collaboration scripts are either represented as written notes on a sheet of paper (e.g., prompt cards in ASK to THINK - TEL WHY), or are re-presented by the teacher repeating them occasionally (e.g., Reciprocal Teaching), or are not externally represented at all (once learners have adequately internalized them during preceding training sessions and during the course of collaboration).

In conclusion, collaboration scripts developed for face-to-face learning focus on encouraging learners to engage in effective collaboration on an individual, cognitive-elaborative level. Learners are supposed to conduct higher-order activities, such as generating elaborated explanations and asking thoughtful questions, for which they often receive highly specific support. Studies by Webb (1989) have shown that generating such explanations can improve knowledge acquisition. The act of questioning leads to a check of the learner's current understanding and ensures better learning of the text (Graesser and Person, 1994). Because collaboration scripts for face-to-face learning aim to foster such high-level (cognitive and metacognitive) learning (King, 1997), the merit of this research tradition lies in its ability to provide insights into the instructional design of what might be termed learning enhancers: Features that increase the learning success of individual learners engaging in collaborative learning to levels they would not be able to reach without instructional support. 
Collaboration scripts for face-to-face learning are often designed in a rather detailed manner. Larger activities are often broken down into smaller substeps, which are also scripted. For example, asking questions in ASK TO THINK - TEL WHY is broken down into asking review questions, probing questions, thinking questions, hint questions, and metacognitive questions. Furthermore, collaboration scripts used for face-to-face learning often (at least in the beginning before the script instructions are internalized by the learners) do not allow the learners much freedom to decide (a) which activities to carry out at a particular point in time and (b) $\underline{\text { how }}$ to conduct these activities. The goal of highly structuring collaborative learning processes is to engage learners in fruitful collaboration processes while setting constraints for engaging in suboptimal ones. As Dillenbourg (2002) describes, such highly specific instructions do, however, bear the danger of over-scripting. Over-scripting implies the loss of freedom and, therefore, may contradict the very nature of collaborative learning as an open, non-coercive endeavor. There seems to be a trade-off between effective structuring (effective in that it supports high-level cognitive and metacognitive processes) and over-scripting, which needs to be considered by designers of collaboration script approaches. The optimum degree of structuredness of a collaboration script is open to future research.

\section{Collaboration Scripts for Computer-Mediated Learning}

We now turn to an analysis of collaboration scripts developed for computer-mediated learning. Due to the dynamic development of networked computer technologies, a number of collaboration scripts for computer-mediated settings have been described over the last few years, starting with the pioneering work of Scardmalia and colleagues on developing the CSILE environment (Scardamalia et al., 1989; Scardamalia and Bereiter, 1991, Scardamalia and Bereiter, 1993/1994). Although the term collaboration script is high on the agenda of the 
research community on Computer-Supported Collaborative Learning (CSCL; e.g., Dillenbourg and Jermann, in press; Dönmez et al., 2005; Ertl et al., 2005; Kollar et al., 2005; Miao et al., 2005; Schellens et al., 2005; Weinberger et al., 2005), not all authors call their approaches collaboration scripts. The selected approaches we present do, however, meet the criterion of supporting collaborative learning through encouraging specific collaboration processes. This selection of approaches reflects the variety of scripts that have been developed in this strand of research. The group size ranges from dyads of learners to a theoretically infinite number of learners. They also cover the most common synchronous and asynchronous communication media like chat tools and discussion boards. Further, all approaches are prominent in their field and underwent empirical research. As can be seen in Table 2, the approaches considered in this article are the ones by Baker and Lund (1997), Hron et al. (1997), Pfister and Mühlpfordt (2002; Learning Protocol Approach) and by Guzdial and Turns (2000; Collaborative and Multimedia Interactive Learning Environment).

\section{INSERT TABLE 2 ABOUT HERE}

The approach by Baker and Lund (1997). This approach integrates a collaboration script into a text-based learning environment in which two learners communicate by aid of a chat tool and a shared physics diagram. The learners' task is to create collaboratively an energy chain model. Learners are provided with a real physical apparatus and a text highlighting the main concepts of the problem. The communication interface includes a number of buttons containing short sentences or sentence starters to guide the learners' interaction. These buttons represent four groups of communicative acts. The first set of buttons deals with the construction of an energy chain in the shared diagram (e.g., "I think that..."), the second represents communicative acts with the aim of reaching an agreement (e.g., "OK"), the third is designed to manage the 
learners' interaction (e.g., "Where do we start?”), and the remaining buttons represent acts geared towards doing something else (e.g., "Read the handout!"). A mouse click on buttons with complete sentences (e.g., "Do you agree?") immediately makes the sentence visible on the partner's screen. A click on a button containing an incomplete sentence (e.g., "I think that...") causes the sentence fragment to be copied into the text window on the learner's own screen. Then, the learner is supposed to complete the sentence and send it to the shared chat window.

With respect to the five conceptual components introduced earlier, the collaboration script developed by Baker and Lund (1997) can be analyzed as follows: The approach has two main objectives. The first objective is to enable learners to understand the energy concept by modeling energy flow behavior. The second objective is to help learners overcome communication problems associated with the characteristics of chat communication, such as incoherent text, missing nonverbal cues, etc. (Schwan, 1997; Fabos and Young, 1999). The activities the learners engage in are also two-fold. First, learners are supposed to engage in elaborative activities, such as giving explanations (which is achieved through the provision of buttons like "I propose to..."). Second, the tool directs learners to perform explicit coordination activities ("Do you agree?"; "Where do we start?", etc.). Although the approach does not explicitly prescribe a detailed sequence for engaging in these activities, the various button prompts provide an implicit method of sequencing. For example, beginning a sequence by clicking on the "Do you agree?" button would not make sense. In contrast, "Where do we start?" is clearly supposed to be used at the beginning of collaboration. With respect to the distribution of collaboration roles, it appears that the approach does not explicitly require that the learners take on roles. Rather, the learners' preferences determine if and how they distribute roles, choose which activities to conduct in 
adopting roles, and maintain or switch roles. With respect to the type of representation, the instructions are represented textually, namely in the form of inscriptions on the buttons.

The approach by Hron et al. (1997). Hron et al. (1997) developed a collaboration script that structures a dyad's interaction within a text-based setting. Learners are provided with erroneous graphical diagrams from the field of biology and asked to correct them using a graphical manipulation tool. First, one of the two learning partners receives a system message requesting an initial proposal for correcting the diagram. This initial suggestion has to be formulated and typed into a text field and sent to the learning partner. By clicking on a button with the title "Do you agree?", the learner asks for agreement from the learning partner. This causes a window to appear on the learning partner's screen that displays a request for signaling agreement or disagreement. If the learner disagrees with the partner's suggestion, he or she formulates a statement including the reasons for disagreement. The other learning partner is then asked to read this statement again and to signal agreement or disagreement. This discourse loop is repeated until an agreement on the partner's suggestion is reached. After that, the diagram is released for further manipulation.

The approach of Hron et al. (1997) can be characterized as follows: The objectives of this collaboration script are, first, to help learners acquire domain-specific knowledge about a welldefined biology problem and, second, to facilitate text-based online communication. To reach these objectives, Hron et al. (1997) engage learners in higher-order activities such as explaining and commenting. Coordinative activities (“Do you agree?”) also play a prominent role in this approach. With respect to explaining and commenting, the script instructions are not detailed. They do not provide learners with specific requirements for creating a good explanation or a fruitful comment. In contrast, the coordinative activity of asking for an agreement is highly 
structured. The interface remains blocked until learners come to an agreement. Sequencing of the learning activities is achieved by the interface design: It specifies that learners first must suggest how to correct the structure of the diagram and then request their partner's agreement. Learners can hardly ignore this imposed sequence because the interface does not allow for a deviation from these activities. Similarly, there is a rather explicit distribution of collaboration roles between the two learning partners, although roles are not explicitly labeled: One learner takes on a composer role and one takes on a commentator role. Learners are required to switch roles after the discourse loop is finished. The script's representation type can be characterized by its reliance on textually coded instructions. These instructions are partially located in the system messages that prompt learners to perform a specific activity and partially in the inscriptions of the prompt buttons. One distinctive characteristic of the script is that not all structuring features are visible and therefore externally represented. For example, turn taking is guided by the design of the communication interface so that only one learner can contribute to the discussion at any given time.

The Learning Protocol approach by Pfister and Mühlpfordt (2002). Pfister and Mühlpfordt (2002) developed a collaboration script for a chat-based learning environment for groups ranging from three to five persons, including one tutor. The learners' task is to discuss topics from geology and philosophy. The communication interface provides three kinds of interactionstructuring methods: First, the interface requires learners to indicate explicitly which message they are referring to. This is achieved by asking them to draw an arrow to the particular message or to a specific part of that message. Second, learners are supposed to specify what kind of message they are about to send to the shared chat window. Thus, a menu appears with a list of three possible statement types to choose from: question, explanation, and comment. Once the 
learners have made these first two selections, they are able to write a message and send it to the shared chat window. Third, the communication interface regulates the sequence according to which learners are supposed to send messages to the shared chat window. To this end, the system provides written information in a separate window concerning whose turn it is at that point in time, while blocking the other learning partners' interfaces. Usually, learners are supposed to send their messages in turn. If a learner categorizes a contribution as a question, the system automatically blocks all learners' chat windows and authorizes only the tutor to respond.

The objectives of the Learning Protocol approach are to (a) learn key concepts from geology and philosophy by discussing them on the basis of introductory texts and (b) improve coordination to achieve a more coherent discussion. Students engage in two basic types of activities: First, learners engage in higher-order activities, such as questioning, explaining, and commenting. Learners must categorize their messages as a question, explanation, or comment. However, they are not provided with information on how to compose a relevant question, explanation, or comment. Second, learners engage in coordinative activities such as drawing arrows from their message to the message they want to refer to. Sequencing is achieved by requiring learners to take turns except when the tutor is asked a question. Despite this sequencing, learners are still free to choose when to compose which of the three possible message types. Although collaboration roles are not explicitly defined, the range of possible roles is restricted to a questioner, an explainer, and a commentator. However, these roles are not explicitly labeled, and learners are not told when to engage in which role. The script's representation type includes different codes: The script contains written information about who is supposed to compose a message at any given point in time. Also, the list of the three possible message types is presented textually. A graphical code (arrow) is used to make the reference to 
other messages explicit. The interface is designed to specify which group member has to send a message and when. This is achieved by simultaneously blocking the other group members' chat windows. However, this component is neither explicitly stated nor externally visible to users.

CaMILE (Guzdial and Turns, 2000). Guzdial and Turns (2000) developed a collaboration script approach for discussion forums among an infinite number of learners. The system operates on three design principles. First, to achieve a high level of transparency, it contains specific discussion management features. For example, for each message, the system displays the type (e.g., "new idea", "rebuttal"), author, and date of origin. Second, CaMILE provides certain facilitation features. For example, before typing a note, learners must specify what type of message they want to create. They can choose between five alternatives: new idea, rebuttal, revision, comment, and question. They can also select whether they want to have one of several prompts pasted into their note for further support (e.g., "I propose to..."). Third, CaMILE contains an anchoring feature. An anchor can be any page on the Web that is of interest to the learners when a particular note in CaMILE includes a link to that Web page. This link can be installed by the instructor or by a learner. That note then represents the beginning (or anchor) of a new thread. For example, a teacher may create a web page containing provocative theses on abortion. By adding a link to the discussion forum, this web page automatically becomes the anchor of a new thread. Every participant who clicks on that link is then directed to the discussion forum.

Concerning the outcomes of collaborative learning, the objectives of the CaMILE approach are not pre-specified. The teacher can lead the discussion in multiple directions, although the script's structure implies that teachers will mainly use the system to support the learners' acquisition of domain-specific knowledge. The main process-related objective is to achieve a 
more coherent discussion by including instructions that encourage learners to focus on a specific topic. The activities supported by CaMILE revolve around elaborative strategies such as explaining, rebutting, or questioning. A distinctive feature is that the learners can choose to paste prompts into their message to support its composition. If a learner does this, the system's support becomes more structured with respect to the chosen activity. With respect to sequencing, CaMILE does not include any features prescribing when to compose what type of message - or even to compose a message at all. Because the script provides learners with a list of prescribed learning activities, it implicitly triggers an engagement in either a composer- or a commentatorlike collaboration role. The same is true with respect to switching collaboration roles, which is not explicitly prescribed but left to the discretion of the learners. With respect to the script's representation type, the script instructions are provided in a textual format. However, the teacher using the script can give further script instructions, possibly in a graphical or an oral format.

\section{Comparing Collaboration Scripts for Computer-Mediated Learning}

As was the case for the face-to-face approaches analyzed above, several commonalities in the design of collaboration scripts for computer-mediated learning can be identified. With regard to their objectives, the presented approaches focus on fostering the acquisition of domainspecific knowledge about the learning material. In the approach by Baker and Lund (1997), for example, learners are expected to acquire knowledge about the energy concept, whereas Hron et al. (1997) focus on helping learners acquire knowledge about the biology problem at hand. All the approaches also emphasize achieving smooth communication and coordination among the learners. In the Learning Protocol approach, for example, this is achieved by designing the communication interface in such a way that it controls turn taking. In CaMILE, communication 
and coordination are supported by requiring learners to label each contribution as a new comment, question, or rebuttal.

Each of the presented approaches aims at supporting slightly different types of activities. In general, two categories of learning activities can be identified: (a) cognitive-elaborative activities (e.g., explaining, commenting) and (b) communicative-coordinative activities (e.g., requesting agreement). However, in all the presented approaches for computer-mediated learning, the script instructions focus more heavily on communication and are specific with respect to each learner's involvement. Hron et al. (1997) require that the learners request their partner's agreement to their own activities. Baker and Lund (1997) provide support for effective communication activities to keep the workflow going. Pfister and Mühlpfordt (2002) are more concerned with promoting higher-order learning activities like explaining and commenting (as compared to the other three approaches), but they also focus on helping learners overcome the limitations associated with chat-based communication. Due to their focus on supporting communication and coordination, the approaches predominately support group processes that occur on a social rather than on an individual level. Individual learning processes are therefore implicitly treated as a consequence of smooth communication and coordination. As an exception, the CaMILE approach focuses more heavily on engaging the individual learner in cognitive-elaborative activities by offering support for activities like explaining or commenting. Yet, learners decide whether to paste prompts into their messages and can circumvent this support. In the Learning Protocol approach, learners are supposed to provide explanations but are not told what a good explanation is. The script does not provide learners with guidance on how to carry out the activities in a way that would relate more closely to individual knowledge construction. Learners may indeed engage more in giving explanations but presumably only learners with high-level explanation abilities 
will benefit, whereas learners with lower-level abilities may fail to create good explanations. Thus, collaboration scripts for computer-mediated learning are not as specific for higher-order, cognitive-elaborative learning activities as for communicative-coordinative activities.

The sequencing of particular learning activities is achieved differently in each of the approaches. The script developed by Baker and Lund (1997) provides a large degree of flexibility concerning when to carry out specific learning activities. The learners basically decide for themselves when to engage in one of the activities. Similarly, the scripts developed by Pfister and Mühlpfordt (2002) and Guzdial and Turns (2000) do not provide learners with clear guidance on when to engage in a particular activity because the learners have the opportunity to choose when they want to generate a specific type of message. The cues presented in the different script approaches do sometimes implicitly trigger certain action sequences. It can be assumed that providing learners with less restrictive collaboration scripts will trigger internal action sequences that guide further collaboration among the learning partners. In contrast, Hron et al. (1997) provide learners with rather explicit guidance concerning the sequence of activities they have to perform. The design of the communication interface stipulates that learners always take turns in giving suggestions or providing feedback to their partner's utterances.

With respect to role distribution, the analysis shows that some scripts for computermediated learning provide clearer specification than others. For example, in the Learning Protocol approach, collaboration roles and the related learning activities are rather explicit (at least in their labels), even though learners are free to choose one of the three induced collaboration roles. However, the communication interface hardly allows for any activities different from the ones that are presented as possible message types. In a similar way, this is also true for the script provided by Hron et al. (1997). In contrast, Baker and Lund's (1997) approach 
does not assign learners to specific collaboration roles. They can choose and design their roles without being bound to them for a whole learning phase. Similarly, CaMILE allows learners to choose whether they want to engage in a composer role to create a new idea, or whether they want to act as a commentator and provide a comment on a learning partner's idea. In the approach by Baker and Lund (1997) and in CaMILE, learners decide for themselves whether to adopt and perform a collaboration role.

The representation type of collaboration scripts for computer-mediated learning is different in each of the presented approaches but may also vary within one particular approach. For example, the Learning Protocol approach uses different formats. The design of the communication interface requires learners to label their contributions as explanation, comment, or question. Learners are required to select the appropriate type from a written list represented in an applet window. In contrast, the learners' task of indicating which message they wish to refer to is represented in a graphical format (in the form of arrows). The third script component, sequencing, is represented textually in a separate window. The other three approaches rely mostly on textual representations of the script instructions. In the Hron et al. (1997) approach, the interface specifies textually which learning partner is supposed to engage in a particular activity at what point in time. CaMILE and the approach presented by Baker and Lund (1997) also rely on textual representations for each of their specific collaboration script components. Some of the presented approaches are characterized by script components that are not visible or otherwise directly perceivable for the learners because they are part of the interface design. For example, in the approach by Hron et al. (1997), sequencing is not externally represented by a list specifying which learner's turn it is. Instead, the system simply blocks the chat window of the learner who is not supposed to write a contribution in the shared chat window. 
In conclusion, the most apparent commonality of the presented collaboration scripts for computer-mediated learning is that all of them support and coordinate communication. Research on collaboration scripts for computer-mediated learning, therefore, provides insights into the instructional design of what might be termed the interactional essentials of collaborative learning: Features of the learning environment that guarantee that the basic interactional requirements for effective collaborative learning processes are met.

Furthermore, some collaboration scripts for computer-mediated learning do not consider detailed instructions for higher-order learning processes as a major determinant of successful collaboration. Quite often, these approaches provide learners with unspecified script instructions and a great deal of freedom concerning how to engage in higher-order activities. Providing rather open collaboration scripts might be adequate for learners who already know how to engage effectively in collaborative learning situations. Problems may arise when learners lack or hold only inadequate knowledge about how to formulate a good explanation, for example. A lack of collaboration abilities might result in a deficient repertoire of effective collaboration strategies, causing learners to make inefficient use of the freedom provided by the collaboration script. These learners are then likely to fail to reach the desired learning objectives. Against this background, we submit that collaboration scripts for computer-mediated learning should also provide learners with more detailed support for how to engage in higher-order learning activities (Rosenshine and Meister, 1994) that are more closely related to individual knowledge acquisition. Initial steps in this direction have, however, already been taken in the selected approaches. For example, the activities in CaMILE (such as analyzing and commenting) are activities that can be viewed as leading to higher-order learning, such as an acquition of elaborative and metacognitive skills. However, Guzdial and Turns (2000) concede that the 
provision of the specific cues in CaMILE "does not, in itself, mean that learning is going on" (p. 441). This indicates that further, more specific instructional support might help. Similarly, the other three approaches remain unclear concerning the question of how learning activities, their sequence, the type of representation, and further features of the presented collaboration scripts relate to higher-order learning gains.

Concerning the type of representation, it appears that collaboration scripts for computermediated settings are often designed rather intuitively. Although all of the approaches presented have been designed innovatively and creatively, there is often a lack of theoretically and empirically guided analysis on how the respective script features should be represented during the learning process. There is research on how learners process different forms of external representations and how these affect learning (e.g., Mayer, 2001; Schnotz, 2002; Suthers and Hundhausen, 2003; Vekiri, 2002). Although this research was mainly conducted with individual learners, it should be considered by developers of collaboration scripts for computer-mediated learning to avoid any learning problems stemming from bad interface design. One real advantage of research on collaboration scripts for computer-mediated learning, however, is that scripts can be imposed without even making them explicit to the learners. In a face-to-face setting, this would hardly be possible. By reducing the amount of instruction, it is likely that learners will experience less cognitive load and have more cognitive resources available to elaborate upon content information (Sweller et al., 1998). This is likely to yield more desirable learning outcomes than when learners have to struggle to understand externally represented instructions. 


\section{What Can Researchers Who Investigate Collaboration Scripts for Face-to-face and for Computer-Mediated Settings Learn from Each Other?}

Our comparison of collaboration script approaches for face-to-face and computer-mediated learning with regard to the five conceptual components introduced at the beginning of this article reveals commonalities and differences -- as well as strengths and weaknesses -- of the two lines of research. Both lines of research can inform researchers investigating collaboration scripts from either perspective about how to design powerful collaboration scripts for various group sizes, tasks, communication media, and learning settings. However, not all problems in one research line are solvable by strengths in the other. Instead, there are also shared deficits. In this section, we delineate commonalities and differences between the two lines of research, as well as deficits that are shared by both of them.

Concerning objectives, collaboration scripts designed for both settings have a strong cognitive focus (e.g., text learning or problem solving). In all face-to-face learning approaches under examination, there was an additional focus on metacognitive objectives, such as acquiring monitoring strategies. Collaboration scripts for computer-mediated learning, in contrast, often exhibit smooth communication and coordination between the collaborators as a secondary objective. To date, neither of the two lines of research has dealt extensively with developing approaches to foster motivational or emotional variables. This is unfortunate because there is already preliminary empirical evidence indicating that motivation can suffer when learners are provided with overly detailed collaboration scripts (e.g., Kollar, 2001).

Both collaboration scripts for face-to-face and computer-mediated learning aim to facilitate cognitive and metacognitive activities, such as explaining, questioning, or commenting. Yet, the 
ways in which these activities are typically introduced differ between the two lines of research. In approaches for computer-mediated learning, activities are often only mentioned so that learners perform them without further instructions. In approaches for face-to-face learning, collaboration scripts often provide additional guidance (either through training or written support) for how to engage in these activities. Collaboration scripts for computer-mediated learning also exhibit a focus on communicative and coordinative activities. This focus is rooted in the different communication characteristics between face-to-face and particular computermediated forms of communication. Computer-mediated collaboration often requires more explicit coordination efforts because nonverbal cues or other opportunities for coordination are limited (e.g., Dillon and Gabbard, 1998; O’Connaill and Whittaker, 1997). Consequently, script instructions concerning communicative-coordinative acts are often highly specific in collaboration scripts for computer-mediated learning.

The sequencing of activities in collaboration script approaches for face-to-face learning is often explicit. Learners receive clear instructions concerning when to engage in an activity. These instructions are introduced by training and often reinforced during collaboration by the teacher or by external artifacts such as prompt cards. In approaches for computer-mediated learning, sequencing is often left up to the learners. However, the communication interface often suggests an implicit sequence for the activities to be performed. For example, learners are given prompts that only make sense when they are used at a particular point in time. Another design strategy used in computer-mediated learning is implicit sequencing through a communication interface that, for example, blocks all chat windows except the window of the learning partner who is supposed to make a contribution. This design feature frees up learner resources for ontopic discussion and learning. 
In many collaboration scripts for face-to-face learning, role distribution is often explicit because the scripts provide detailed instructions concerning which learner has to act in which role at what specific point in time. In contrast, approaches for computer-mediated learning often distribute collaboration roles in a rather implicit manner. The actual design of the communication interface might, however, suggest the specific roles learners should perform. For example, a communication interface can offer sentence starters that prompt learners to assume the role of an explainer or a questioner.

Concerning the representation type, most approaches for face-to-face learning use textual (e.g., prompt cards) and auditive representations (e.g., the teacher explaining the specific instructions). However, most of the script instructions are represented mentally because learners are supposed to internalize them before collaboration. In collaboration script approaches for computer-mediated learning, instructions are often represented textually or graphically. In some cases, the interface is designed to only allow for one specific activity or sequence to be carried out without actually representing that script component externally.

As we have argued, collaboration scripts for computer-mediated learning typically focus on facilitating communicative and coordinative processes. These are processes that operate on the intersubjective level: By focusing on communication and coordination, the primary targets of the script instructions are the interactions among the group members rather than the cognitive processes of each individual learner. Thus, research on collaboration scripts for computermediated learning generates knowledge about interactional essentials, i.e., how to structure learning environments that enable learners' smooth interaction. Research on collaboration scripts for face-to-face learning, on the other hand, supports learners to engage in individual elaborative activities. The main focus of collaboration script research for face-to-face learning, therefore, are 
the learning enhancers of collaborative learning, i.e., how to design scripts that enable learners to deeply elaborate content information. It can be claimed that better collaboration script approaches can be developed in the future when insights from the two lines of research come together. The result would be a development of approaches that guarantee smooth communication and coordination, as well as higher-order individual learning (Rosenshine and Meister, 1994).

The differences between collaboration script research for computer-mediated vs. face-toface learning are also reflected on a theoretical level. Authors of collaboration script approaches for face-to-face learning often refer to cognitive learning theories and information-processing models. These approaches regard knowledge construction as a process in which an individual integrates incoming information with pre-existing knowledge structures. In contrast, research on collaboration scripts for computer-mediated learning often refers to sociocultural (Vygotsky, 1978) and situated theories (e.g., Lave and Wenger, 1991) of cognition. Sociocultural and situated approaches emphasize the importance of social practices in a community of learners. As a consequence, collaborative learning environments should be designed to guarantee that individuals can fruitfully participate in these practices. Supporting participation in group activities and enhancing individual cognitive processes is, however, not contradictory. Both can be reached with a well-designed collaboration script that supports each of four poles - supporting groups as well as individuals on the one hand and supporting communication as well as elaboration on the other. Learners may be provided with multiple scripts that are directed towards different objectives and activities. There is a danger, however, of providing learners with

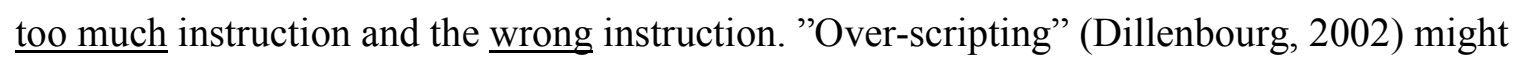
prove more detrimental for some learners than for others. For example, adults might have 
developed patterns of collaboration that are highly specific and useful for specific situations. Imposing too much structure is likely to produce reactance (Brehm, 1966) and motivation loss (Kollar, 2001). For adult learners, one solution could be to design collaboration scripts that allow them to rely on their own experiences and strategies to structure their collaboration processes.

Correspondingly, research has not yet focused on the question of how structured a collaboration script should be to pursue its specific learning objectives. Dillenbourg (2002) and Cohen (1994) point to the dangers of micro-structuring processes of collaborative learning, especially in sophisticated tasks that require creative problem solutions. In such tasks, highly structured collaboration scripts can reduce the learner's freedom and limit high-level discourse. Furthermore, different groups or types of learners may require differently structured collaboration scripts. One critical learner characteristic might be domain-specific prior knowledge (Dochy et al., 1999). Learners with low domain-specific knowledge might learn better with highly structured collaboration scripts, whereas learners with high domain-specific knowledge may require collaboration scripts that are more open. In addition, it is reasonable to assume that the success of collaborative learning is also affected by learners' domain-general prior knowledge that guides them in collaborative settings (Kollar et al., 2005). Research needs to examine what specific collaboration knowledge individuals bring to a collaborative learning situation and whether an externally provided collaboration script activates adequate knowledge or compensates for deficient knowledge. We view this interplay of individuals' prior knowledge about collaboration and externally provided collaboration scripts as a core question for both the design of collaboration scripts and future theory building. Therefore, we develop a conceptual framework on learning with collaboration scripts that includes the learners' domain-general knowledge on collaboration. This framework is presented in the last section of this article. 


\section{A Person-Plus Framework of Collaboration Scripts: Future Directions for Theory Building and Empirical Research}

Although research on collaboration scripts originally derived the term script from cognitive psychology (Schank and Abelson, 1977), most researchers departed from the individualistic notion of scripts as internal memory structures. Maybe as a consequence, research on collaboration scripts for face-to-face and for computer-mediated learning has largely neglected the importance of the individual and his or her procedural knowledge that guides behavior in collaborative situations. In collaboration script approaches that are less highly structured, however, effective collaboration strongly depends on how the learning partners structure their collaboration. According to Schank and Abelson (1977), individuals develop internal knowledge structures or scripts through repeated participation in particular situations. These structures or scripts guide their understandings and actions within these situations. Applied to collaborative learning, cognitive scripts about how to engage in collaboration develop as an individual repeatedly engages in collaborative situations beginning in early childhood. This domain-general knowledge about collaboration may be termed internal collaboration scripts. It can be argued that the structure of the learners' internal collaboration scripts is one central determinant of collaborative learning and that it interacts with the structure of an externally provided collaboration script (see Carmien et al., in press).

We derive valuable ideas from theoretical approaches on distributed cognition to conceptualize the interplay between internal and external collaboration scripts (e.g., Derry et al., 1998; Hewitt and Scardamalia, 1998; King, 1998; Moore and Rocklin, 1998; Pea, 1993; Perkins, 1993; Salomon, 1993). In his person-plus-surround concept, Perkins' (1993) distinguishes between the person-solo and the person-plus; both are involved when an individual is asked to 
solve a task in conjunction with other persons and/or an external artifact (e.g., a collaboration script). The person-solo describes the individual as one component of the system. The personplus describes the whole system that comprises both the individual and his or her social and artifactual surround. In this way, cognition or "intelligence" (Pea, 1993) is viewed as distributed between individuals and artifacts, thereby challenging the traditional cognitive stance that knowledge and intelligence is solely represented "within the head" of an individual (cf. Anderson, 2000). Although the notion of distributed cognition that Perkins (1993) advocates is not without criticism (e.g., Moore and Rocklin, 1998; Newell, 1990), it can help conceptualize the interplay between internal and external collaboration scripts.

When accepting the notion that knowledge can be distributed between a person-solo and the surround, it can be asked where the knowledge necessary for task accomplishment actually is located - in the cognitive system of the individual or in the surround. For Perkins (1993), however, this question is secondary. More important are the access characteristics of that knowledge (i.e., how easily this knowledge is accessible for the person-plus system; Perkins, 1993). For the system's task performance, no qualitative difference is assumed when the necessary task-relevant knowledge is located in the person-solo or in the surround. For Perkins, what is more important than the question concerning the location of task-relevant knowledge within the person-plus system is the question concerning what system component has metacognitive control over the system (executive function; Perkins, 1993). This function can be adopted by either the person-solo (e.g., a learner who sets learning goals and monitors his or her individual progress and the group process) or the surround (e.g., an external collaboration script that sets rules and goals and monitors their accomplishment). In other cases, the surround might 
support the individual in adopting the executive function over the system.In this case, both internal and external scripts contribute to controlling the system.

Perkins' (1993) conception is helpful in understanding how groups of learners engage in collaboration tasks because it includes both the individual learners and their specific knowledge about collaboration and external support systems, such as collaboration scripts, as contributors to the collaborative activity (see also King, 1998). By adopting a person-plus view, the previous components of our analysis can be expanded by using core concepts of Perkins' person-plus approach (e.g., accessibility of knowledge; executive function). The resulting conceptual framework takes the contributions of internal and external collaboration scripts into account. This framework (Table 3) can be useful for integrating and stimulating research on collaborative learning with collaboration scripts.

\section{INSERT TABLE 3 ABOUT HERE}

To develop a person-plus framework of collaboration scripts, it is useful to first determine the main factors involved when a group of learners tries to accomplish a collaboration task. On a general level, at least three factors can be distinguished: (a) a global collaborative activity (or a set of global activities) the system needs to perform to solve the task, (b) knowledge about collaboration that is required to conduct these activities, and (c) the system's executive function that sets the goals and monitors the processes of collaboration. When mapping the five conceptual components of collaboration scripts identified earlier onto these three main factors, the following picture of collaboration can be drawn:

The activity learners are engaged in can initially be described in terms of the main objective that underlies it. For example, as in the approach by Baker and Lund (1997), the activity's objective might be to construct collaboratively a model of energy flow. The whole 
activity might then be broken down into several sub-activities needed to pursue that goal. In the Baker and Lund script, these activities include "give a proposal to change the energy chain model", or "ask for your partner's agreement". These activities might need to occur in a specific sequence. Applied to the learning scenario in the Baker and Lund approach, the script might instruct the learners to start by providing a proposal on how to change the current model. Then, the learner can ask his or her learning partner if the proposal is acceptable and change the energy chain model accordingly. In addition, the sub-activities might be attached to collaboration roles such as a problem-solver and a commentator.

The knowledge dimension should include at least two sub-categories. First, knowledge about how to act in collaboration tasks can be characterized by the representation type, which was one of the five conceptual script component introduced earlier. The type of representation can include different mental, as well as graphical, oral, or written representations. For example, a learner may hold a mental representation of collaboration that guides him or her to first discuss how to approach the task. In another case, instructions on a sheet of paper may explicitly state how the learning partners should act to pursue the goal of collaboration. Perkins' (1993) personplus perspective suggests that knowledge about collaboration can also be characterized with respect to its accessibility characteristics. Accessibility is presumably a central determinant of successful task accomplishment. If the knowledge about collaboration that is required to solve the collaboration task is not accessible in either the internal or the external collaboration script, then a system consisting of two or more individuals and an external collaboration script will fail to accomplish the collaboration task. If the knowledge is represented in the external collaboration script, then learners can use it to guide their activities and solve the collaboration task. If one of 
the collaborators has the required knowledge accessible in his or her internal script, then this internal knowledge might translate more quickly into appropriate collaborative action.

With respect to the executive function, an analysis has to focus on (a) how the planning and control of goal setting and (b) the planning and control of performance is achieved. Goal

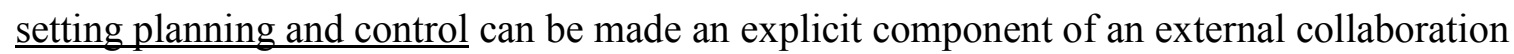
script ("Create an energy chain model!"). Goal setting may also be less specified in the external collaboration script and be transferred to the learners themselves (e.g., when learners are given the opportunity to set their own goals, such as "Choose a hypothesis you would like to defend in class"). Performance planning and control can similarly be either specific or more open. The Hron et al. approach, for example, can be regarded as specific because the modeling window is blocked so long as partners do not find an agreement on the next solution step. Reciprocal Teaching, in contrast, can be regarded as open with respect to performance planning and control. For example, the teacher asks learners to be aware of specific communication skills, which, in the end, are largely subject to the learners' internal collaboration scripts.

\section{Potential of a Person-Plus Framework of Collaboration Scripts}

What are the areas of potential for a person-plus framework of collaboration scripts? First, when there is acknowledgement that collaborative learning processes are partially guided by internal scripts, partially by external scripts, and partially by their interaction, the resulting framework can help define differences among existing collaboration script approaches more clearly. We assume that specific aspects of interactions between learners, such as their sequencing behavior or their engagement in particular activities, are a function of the interplay between their internal scripts and the external collaboration script. For example, CaMILE induces sub-activities, such as generating a new idea or commenting on an existing idea, in an 
explicit manner by providing learners with a limited selection of possible message types to choose from. This means that sub-activities are largely determined by the external collaboration script. In contrast, in the sequencing dimension, CaMILE provides learners with a great deal of freedom to decide when to engage in which sub-activities. Thus, in the sequencing dimension, collaboration in a CaMILE scenario is largely driven by the learners' internal collaboration scripts. As another example, the ASK to THINK - TEL WHY script specifies sub-activities such as asking probing questions or giving explanations. In contrast, performance planning and control depends on the teacher or on the learners themselves.

In general, relating different collaboration script approaches to the dimensions of the person-plus framework of collaboration scripts might support a more thorough interpretation of the often inconsistent findings concerning the effects of external collaboration scripts. One outcome could be that external collaboration scripts that strongly guide the learners' subactivities are only effective for specific types of learners, whereas a detailed prescription of specific sequences would be detrimental for them.

Second, the person-plus framework of collaboration scripts can be used as a guideline for designing external collaboration script approaches. By explicitly acknowledging the importance of learners' internal collaboration scripts, the person-plus framework encourages designers to consider the characteristics and specific needs of the actual users working with the collaboration script. However, the learners' internal collaboration scripts need to be assessed in a reliable way. Therefore, adequate instruments need to be developed.

Third, the person-plus framework of collaboration scripts can provide an opportunity to align research efforts in the field more effectively. As the variety of collaboration script approaches presented in this article indicates, research on collaboration scripts is diverse, making 
it hard to integrate the different results obtained in the numerous empirical studies on the topic. With the person-plus framework presented, it should prove easier to approach and identify systematically open and pressing research questions. In our view, one of the most important research needs relates to the question of how and what collaboration script information should be distributed between the person-solo and the surround (see King, 1998). According to Perkins (1993), knowledge about higher-level processes should be kept in the person-solo (e.g., create arguments), whereas lower-level processes should be shifted to the surround (e.g., performing an addition equation by using a hand calculator). Instructions concerning low-level operations like "Now click on the OK-button" or "Wait until the diagram is released for further manipulation" might neither be part of the learners' internal scripts nor be a relevant objective for internalization. On the other hand, at certain stages of collaboration, it might be beneficial to omit certain aspects of the external script. One can assume that learners, through interaction with the external script, develop and constantly refine knowledge about how to structure their collaboration, thereby gradually integrating procedures represented in the external collaboration script into their internal scripts.

Finally, future research should address and investigate the dynamics of the interplay between internal and external collaboration scripts (Kollar et al., 2005). There are at least three different patterns of how internal and external collaboration scripts might interact. One possibility is that external collaboration scripts supplant internal scripts. This might be the case when an external collaboration script does not allow for any task procedures other than the ones intended by its designer. A second possibility is that external and internal collaboration scripts have additive effects, such that external scripts trigger existing internal collaboration scripts that would not be activated without external support. Thus, a system including scripts located in the 
person-solos and the surround would be superior to a system in which learners only have their person-solo collaboration scripts available. A third possibility is that there are interactive effects between internal and external collaboration scripts. It is reasonable to assume that as learners interact more and more with external collaboration scripts, a gradual internalization of script contents takes place. As learners internalize these external script contents more and more, the specificity of the external script would need to gradually be reduced to ensure that learners are not given instruction they actually do not need. The process of reducing the amount of external instruction is known as fading (see Pea, 2004). Effective fading requires sophisticated methods for online assessment. The interaction patterns that occur between the learners of a group must be assessed to obtain a clear picture of which portions of the external collaboration script should be faded out when learners are diagnosed as having internalized them. In environments for Computer-Supported Collaborative Learning (CSCL), this could be increasingly achieved through language analysis tools implemented in the system that record the learners' interaction and that use these data to adjust the structuredness of an external collaboration script accordingly. Dönmez et al. (2005) showed that after training, algorithms developed in the field of applied linguistics can analyze online discussions on a specific topic with reliabilities comparable to independent human coders. Adaptive external scripts could be developed if the results of these automated analyses were fed back into the design of the external collaboration script. The findings would then lead to fading specific components in or out as appropriate. In face-to-face learning, this assessment has always been the task of the teacher and is based on his or her observations of a group's learning processes. For classroom settings, using computersupported online knowledge assessment techniques as developed by Dönmez et al. (2005) might be a promising way to support teachers in their evaluatory efforts and in their decisions about the 
structure of the collaboration script required by a specific group. This way, collaboration scripts provided by a computer and scripts provided by a teacher can combine to provide optimum support for collaborating groups - an instance Tabak (2004) calls synergistic scaffolding. 


\section{Acknowledgements}

This research has been partially funded by the Deutsche Forschungsgemeinschaft (DFG;

[German Research Foundation]). 


\section{References}

Anderson, J. R. (2000). Cognitive psychology and its implications (Fifth edition), Worth Publishing, New York.

Baker, M., and Lund, K. (1997). Promoting reflective interactions in a CSCL environment. $J$. Computer Assisted Learning 13: 175-193.

Bell, P. (2004). Promoting students' argument construction and collaborative debate in the science classroom. In Linn, M. C., Davis, E. A., and Bell, P. (eds.), Internet environments for science education, Erlbaum, Mahwah, NJ, pp.114-144.

Brehm, J. W. (1966). A theory of psychological reactance, Academic Press, New York.

Carmien, S., Kollar, I., Fischer, F., and Fischer, G. (in press). The interplay of internal and external scripts. A distributed cognition perspective. To appear in Fischer, F., Mandl, H., Haake, J., and Kollar, I. (eds.), Scripting computer-supported collaborative learning: Cognitive, computational, and educational perspectives, Springer, New York.

Cohen, E. G. (1994). Restructuring the classroom: Conditions for productive small groups. Rev. Educational Research 64(1): 1-15.

Cohen, E. G., and Lotan, R. A. (1995). Producing equal-status interaction in the heterogeneous classroom. Am. Educational Research J. 32: 99-120.

Derry, S. J., DuRussel, L. A., and O’Donnell, A. M. (1998). Individual and distributed cognitions in interdisciplinary teamwork: A developing case study and emerging theory. Educational Psychology Rev. 10(1): 25-56.

Dillenbourg, P. (2002). Over-scripting CSCL: The risks of blending collaborative learning with instructional design. In Kirschner, P. A. (ed.), Three worlds of CSCL. Can we support CSCL, Open Universiteit Nederland, Heerlen, pp. 61-91.

Dillenbourg, P., and Jermann, P. (in press). Designing integrative scripts. To appear in Fischer, 
F., Mandl, H., Haake, J., and Kollar, I. (eds.), Scripting computer-supported collaborative learning: Cognitive, computational, and educational perspectives, Springer, New York.

Dillon, A., and Gabbard, R. (1998). Hypermedia as an educational technology: A review of the quantitative research literature on learner comprehension, control and style. Rev. Educational Research 68: 322-349.

Dochy, F., Moerkerke, G., and Segers, M. (1999). The effect of prior knowledge on learning in educational practice: Studies using prior knowledge state assessment. Evaluation \& Research in Education 8: 345-367.

Dönmez, P., Rosé, C. P., Stegmann, K., Weinberger, A., and Fischer, F. (2005). Supporting CSCL with automatic corpus analysis technology. In Koschmann, T., Suthers, D., and Chan, T. -W. (eds.), Computer Supported Collaborative Learning 2005: The Next 10 Years, Lawrence Erlbaum, Mahwah, NJ, pp. 125-134.

Ertl, B., Fischer, F., and Mandl, H. (in press). Conceptual and socio-cognitive support for collaborative learning in videoconferencing environments. Computers \& Education.

Ertl, B., Kopp, B., and Mandl, H. (2005). Effects of an individual's prior knowledge on collaborative knowledge construction and individual learning outcomes in videoconferencing. In Koschmann, T., Suthers, D., and Chan, T. -W. (eds.), Computer Supported Collaborative Learning 2005: The Next 10 Years, Lawrence Erlbaum, Mahwah, NJ, pp. 145-154.

Fabos, B., and Young, M. (1999). Telecommunication in the Classroom: Rhetoric versus Reality. Rev. Educational Research 69(3): 217-260.

Fischer, F., Bruhn, J., Gräsel, C., and Mandl, H. (2002). Fostering collaborative knowledge construction with visualisation tools. Learning and Instruction 12: 213-232. 
Graesser, A. C., and Person, N. K. (1994). Question asking through tutoring. Am. Educational Research J. 31: 104-137.

Guzdial, M., and Turns, J. (2000). Effective discussion through a computer-mediated anchored forum. J. Learning Sciences 9(4): 437-469.

Hewitt, J., and Scardamalia, M. (1998). Design principles for distributed knowledge building processes. Educational Psychology Rev. 10(1): 75-96.

Hron, A., Hesse, F. W., Reinhard, P., and Picard, E. (1997). Strukturierte Kooperation beim computerunterstützten kollaborativen Lernen [Structured collaboration in computersupported collaborative learning]. Unterrichtswissenschaft 25: 56-69.

Hythecker, V. I., Dansereau, D. F., and Rocklin, T. R. (1988). An analysis of the processes influencing the structured dyadic learning environment. Educational Psychologist 23(1): $23-37$

Johnson, D. W., and Johnson, R. T. (1994). Constructive conflict in schools. J. Social Issues 50(1): 117-137.

King, A. (1997). ASK to THINK - TEL WHY®C: A model of transactive peer tutoring for scaffolding higher level complex learning. Educational Psychologist 32(4): 221-235.

King, A. (1998). Transactive peer tutoring: Distributing cognition and metacognition. Educational Psychology Rev. 10: 57-74.

King, A. (2002). Structuring peer interaction to promote high-level cognitive processing. Theory into Practice 41(1): 33-39.

Kollar, I. (2001). Gewissheits- und Ungewissheitsorientierung beim kooperativen Lernen in Videokonferenzen - der Einfluss verschiedener Strukturierungsmaßnahmen. [Uncertainty orientation in video-mediated cooperative learning - effects of different structural 
supports]. Unpublished master thesis, Ludwig-Maximilians-University, Munich, Germany.

Kollar, I., Fischer, F., and Slotta, J. D. (2005). Internal and external collaboration scripts in webbased science learning at schools. In Koschmann, T., Suthers, D., and Chan, T. -W. (eds.), Computer Supported Collaborative Learning 2005: The Next 10 Years, Lawrence Erlbaum, Mahwah, NJ, pp. 331-340.

Lambiotte, J. G., Dansereau, D. F., O’Donnell, A. M., Young, M. D., Skaggs, L. P., Hall, R. P., and Rocklin, T. R. (1987). Manipulating cooperative scripts for teaching and learning. J. of Educational Psychology 79(4): 424-430.

Larson, C. O., Dansereau, D. F., O’Donnell, A., Hythecker, V., Lambiotte, J. G., and Rocklin, T. R. (1984). Verbal ability and cooperative learning: Transfer of effects. J. of Reading Behavior 16(4): 289-295.

Larson, C. O., Dansereau, D. F., O’Donnell, A., Hythecker, V., Lambiotte, J. G., and Rocklin, T. R. (1985). Effects of metacognitive and elaborative activity on cooperative learning and transfer. Contemporary Educational Psychology 10, 342-348.

Lave, J., and Wenger, E. (1991). Situated learning: Legitimate peripheral participation, Cambridge University Press, Cambridge.

Mayer, R. E. (2001). Multimedia learning. Cambridge University Press, Cambridge, MA.

Miao, Y., Hoeksema, K., Hoppe, H. U., and Harrer, A. (2005). CSCL Scripts: Modelling features and potential use. In Koschmann, T., Suthers, D., and Chan, T. -W. (eds.), Computer Supported Collaborative Learning 2005: The Next 10 Years, Lawrence Erlbaum, Mahwah, NJ, pp. 423-432.

Moore, J. L., and Rocklin, T. R. (1998). The distribution of distributed cognition: Multiple interpretations and uses. Educational Psychology Rev. 10(1): 97-113. 
Newell, A. (1990). Unified theories of cognition, Harvard University Press, Cambridge, MA.

Nuthall, G. (2000). The role of memory in the acquisition and retention of knowledge in science and social studies units. Cognition and Instruction 18(1): 83-139.

O’Connaill, B., and Whittaker, S. (1997). Characterizing, predicting, and measuring videomediated communication: A conversational approach. In Finn, K. E., Sellen, A. J., and Wilbur, S. B. (eds.), Video-mediated communication, Erlbaum, Mahwah, NJ, pp. 107-132.

O’Donnell, A. M. (1999). Structuring dyadic interaction through scripted cooperation. In O’Donnell, A. M., and King, A. (eds.), Cognitive perspectives on peer learning, Erlbaum, Mahwah, NJ, pp. 179-196.

O’Donnell, A. M., and Dansereau, D. F. (1992). Scripted Cooperation in student dyads: A method for analyzing and enhancing academic learning and performance. In HertzLazarowitz, R., and Miller, N. (eds.), Interaction in cooperative groups: The theoretical anatomy of group learning, Cambridge University Press, New York, pp. 120-141.

O’Donnell, A. M., Dansereau, D. F., Hall, R. H., and Rocklin, T. R. (1987). Cognitive, social/affective, and metacognitive outcomes of scripted cooperative learning. $J$. Educational Psychology 79(4): 431-437.

Palincsar, A. S., and Brown, A. L. (1984). Reciprocal teaching of comprehension-fostering and comprehension-monitoring activities. Cognition and Instruction 1: 117-175.

Pea, R. D. (1993). Practices of distributed intelligence and design for education. In Salomon, G. (ed.), Distributed cognitions: psychological and educational considerations, Cambridge University Press, Cambridge, pp. 47-87.

Pea, R. D. (2004). The social and technological dimensions of scaffolding and related theoretical concepts for learning, education, and human activity. J. Learning Sciences, 13(3), 423-451. 
Perkins, D. N. (1993). Person-plus: a distributed view of thinking and learning. In Salomon, G. (ed.), Distributed cognitions: psychological and educational considerations, Cambridge University Press, Cambridge, pp. 88-110.

Pfister, H.-R., and Mühlpfordt, M. (2002). Supporting discourse in a synchronous learning environment: The learning protocol approach. In Stahl, G. (ed.), Proceedings of the Conference on Computer Supported Collaborative Learning (CSCL) 2002, Erlbaum, Hillsdale, pp. 581-589.

Plass, J. L., Chun, D., Mayer, R. E., and Leutner, D. (1998). Supporting visualizer and verbalizer learning preferences in a second language multimedia learning environment. $J$. Educational Psychology 90: 25-36.

Quintana, C., Reiser, B. J., Davis, E. A., Krajcik, J., Fretz, E., Duncan, R. G., Kyza, E., Edelson, D., and Soloway, E. (2004). A scaffolding design framework for software to support science inquiry. J. Learning Sciences 13(3): 337-387.

Reiser, B. J. (2004). Scaffolding complex learning: The mechanisms of structuring and problematizing student work. J. Learning Sciences 13(3): 273-304.

Renkl, A., Mandl, H., and Gruber, H. (1996). Inert knowledge: Analyses and remedies. Educational Psychologist 31: 115-121.

Rewey, K. L., Dansereau, D. F., Dees, S. M., Skaggs, L. P., and Pitre, U. (1992). Scripted cooperation and knowledge map supplements: Effects of the recall of biological and statistical information. J. Experimental Education 60(2): 93-107.

Rosenshine, B., and Meister, C. (1994). Reciprocal teaching: A review of the research. Rev. Educational Research 64(4): 479-530.

Rummel, N., and Spada, H. (2005). Learning to collaborate: an instructional approach to 
promoting collaborative problem solving in computer-mediated settings. J. Learning Sciences 14(2): 201-241.

Salomon, G. (1993). No distribution without individuals' cognition: A dynamic interactional view. In Salomon, G. (ed.), Distributed cognitions: Psychological and educational considerations, Cambridge University Press, New York City, pp. 111-138.

Scardamalia, M., and Bereiter, C. (1991). Higher Levels of Agency for Children in Knowledge Building: A Challenge for the Design of New Knowledge Media. J. Learning Sciences 1(1): $37-68$

Scardamalia, M., and Bereiter, C. (1993/1994). Computer-support for knowledge-building communities. J. Learning Sciences 3(3): 265-283.

Scardamalia, M., Bereiter, C., McLean, R. S., Swallow, J., and Woodruff, E. (1989). Computer supported intentional learning environments. J. Educational Computing Research 5: 51-68.

Schank, R. C. (1999). Dynamic memory revisited. Cambridge University Press, Cambridge.

Schank, R. C., and Abelson, R. P. (1977). Scripts, plans, goals and understanding, Erlbaum, Hillsdale, NJ.

Schellens, T., van Keer, H., Valcke, M., and deWever, B. (2005). The impact of role assignment as scripting tool on knowledge construction in asynchronous discussion groups. In Koschmann, T., Suthers, D., and Chan, T. -W. (eds.), Computer Supported Collaborative Learning 2005: The Next 10 Years, Lawrence Erlbaum, Mahwah, NJ, pp. 557-566.

Schnotz, W. (2002). Towards an integrated view of learning from text and visual displays. Educational Psychology Rev. 14(2): 101-120.

Schwan, S. (1997). Media characteristics and knowledge acquisition in computer conferencing. European Psychologist 2(3): 277-285. 
Sherin, B., Reiser, B. J., and Edelson, C. (2004). Scaffolding analysis: Extending the scaffolding metaphor to learning artifacts. J. Learning Sciences: 13(3): 387-421.

Spiro, R. J., Feltovich, P. J., Jacobson, M. J., and Coulson, R. L. (1991). Cognitive flexibility, constructivism, and hypertext: Random access instruction for advanced knowledge acquisition in ill-structured domains. Educational Technology 31(5): 24-33.

Suthers, D. D., and Hundhausen, C. D. (2003). An experimental study of the effects of representational guidance on collaborative learning processes. J. Learning Sciences, 12(2): 183-219.

Sweller, J., Van Merrienboer, J., and Paas, F. (1998). Cognitive architecture and instructional design. Educational Psychology Rev. 10 (3): 251-296.

Tabak, I. (2004). Synergy: A complement to emerging patterns of distributed scaffolding. $J$. Learning Sciences 13(3): 305-335.

Vekiri, I. (2002). What is the value of graphical displays in learning? Educational Psychology Rev. 14(3): 261-312.

Vygotsky, L. S. (1978). Mind and society: The development of higher mental processes, Harvard University Press, Cambridge, MA.

Vygotsky, L. S. (1992). Thought and language (rev. ed.). The MIT Press, Cambridge, MA.

Webb, N. M. (1989). Peer interaction and learning in small groups. Int. J. Educational Research 13: 21-39.

Weinberger, A., Ertl, B., Fischer, F., and Mandl, H. (2005). Epistemic and social scripts in computer-supported collaborative learning. Instructional Science 33(1): 1-30.

Wood, D., Bruner, J. S., and Ross, G. (1976). The role of tutoring in problem solving. J. Child Psychology and Psychiatry and Allied Disciplines 17: 89-100. 
Table 1

$\underline{\text { Central conceptual components of collaboration scripts. }}$

Central conceptual components of collaboration scripts

Learning objectives

Type of activities

Sequencing features

Role distribution

Type of representation 
Table 2

Collaboration script approaches for face-to-face vs. computer-learning included in the analysis.

\begin{tabular}{cc}
\hline Learning setting & Approaches included in the analysis \\
\hline Face-to-face & O'Donnell and Dansereau (1992) \\
King (1997) & Johnson and Johnson (1994) \\
Palincsar and Brown (1984) \\
Baker and Lund (1997) \\
Hron, Hesse, Reinhard and Picard (1997) \\
Pfister and Mühlpfordt (2002) \\
Guzdial and Turns (2000) \\
\hline
\end{tabular}


Table 3

Main factors and subcategories involved in a collaborative learning group solving a task.

\begin{tabular}{cc}
\hline Main factor & \multicolumn{1}{c}{ Subcategories } \\
\hline \multirow{2}{*}{ Activity } & Objective of activity \\
\cline { 2 - 2 } & Type of sub-activities \\
\cline { 2 - 2 } & Sequencing of sub-activities \\
\hline Knowledge & Type of representation \\
\cline { 2 - 2 } Executive function & Accessibility characteristics \\
\hline & Goal setting control \\
\hline
\end{tabular}

